\title{
Evaluación de desempeño del algoritmo de seguimiento de características faciales basado en modelos ASM usando Kinect
}

Performance evaluation of a face tracking algorithm based on ASM models using the Kinect Sensor

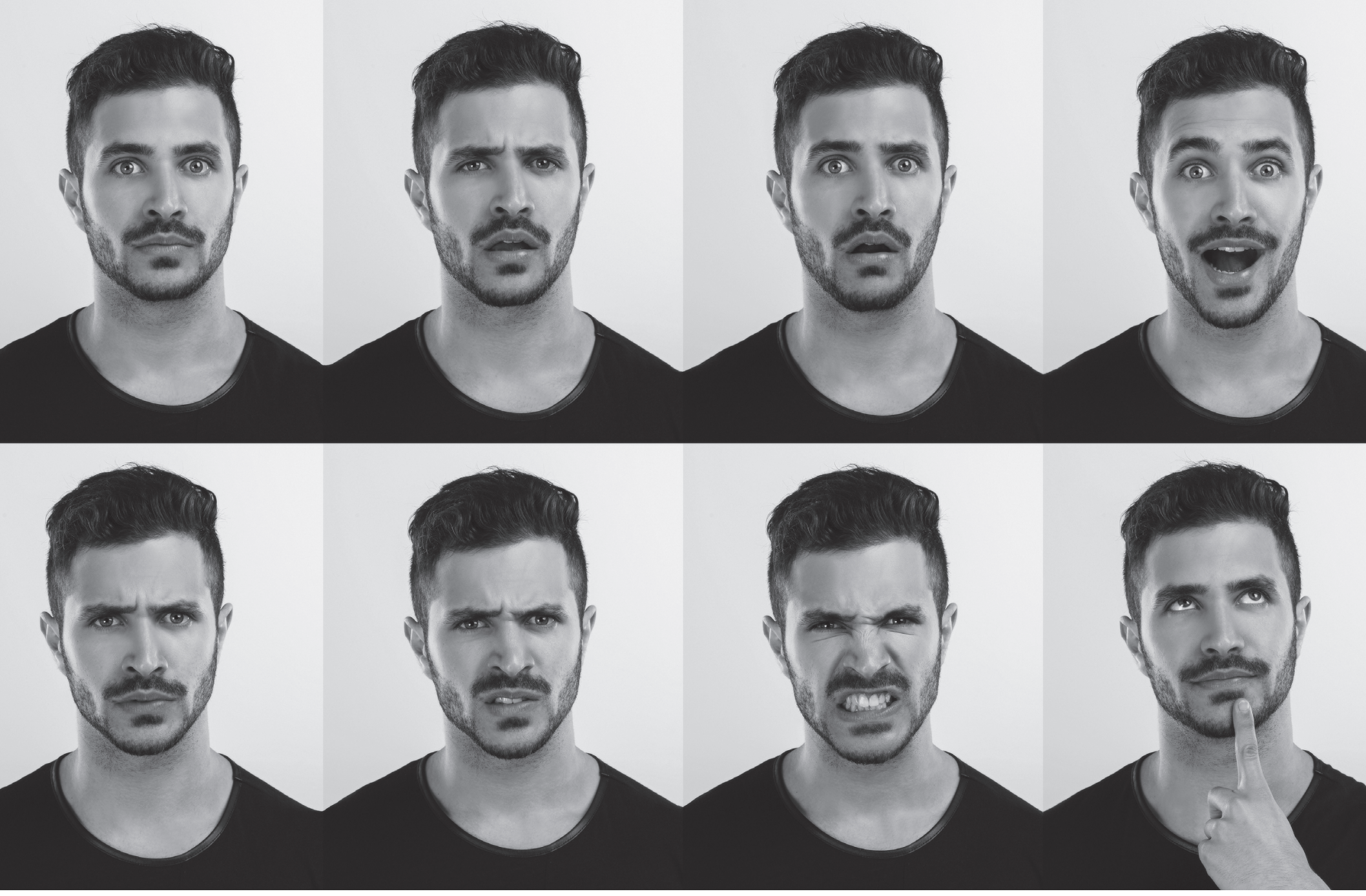




\title{
Evaluación de desempeño del algoritmo de seguimiento de características faciales basado en modelos ASM usando Kinect ${ }^{1}$
}

\section{Performance evaluation of a face tracking algorithm based on ASM models using the Kinect Sensor}

\author{
Diego Alejandro Rodríguez Ardila², Jahir Joel García Mendoza ${ }^{3}$, \\ Carlos Humberto Esparza Franco ${ }^{4}$, Edwin Alberto Silva Cruz ${ }^{5}$ \\ 2,3,4Unidades Tecnológicas de Santander - UTS, Bucaramanga, Colombia. \\ ${ }^{5}$ Universidad de Investigación y Desarrollo, Bucaramanga, Colombia.
}

Artículo recibido en enero de 2017; artículo aceptado en marzo de 2017

\begin{abstract}
Citación del artículo: Rodríguez, D., García, J., Esparza, C. \& Silva, E. (2016). Evaluación de desempeño del algoritmo de seguimiento de características faciales basado en modelos ASM usando Kinect. I+D Revista de Investigaciones,
\end{abstract}

10(2) julio - diciembre de 2017, pp. 80 - 88

\begin{abstract}
Resumen
En este trabajo se presenta la evaluación del desempeño de un algoritmo de seguimiento de las características faciales aplicando modelos de forma activa (ASM) y utilizando el sensor Kinect como dispositivo de captación de imagen. El desarrollo se realizó mediante las librerías de OpenCV, en un PC portátil con procesador Core i5 a 2.4Ghz, 4 Gigabytes de memoria RAM, que corre bajo sistema operativo Windows 7. Para la evaluación se ejecutó el algoritmo para observar la respuesta respecto a las distintas posturas y expresiones faciales. Se tomaron los tiempos de estabilización de los puntos sobre la imagen y se analizó punto a punto y con criterio humano la localización de los puntos sobre la imagen. Para facilitar el análisis se agruparon los puntos acordes a la zona del rostro: contorno de la cara, cejas, nariz, ojos y boca. Por último, se presentan los resultados del tiempo promedio de ajuste del modelo, el promedio de frames, así como un error promedio de posicionamiento en las distintas condiciones del rostro, lo cual muestra la robustez de este trabajo y la adaptabilidad para trabajos futuros.
\end{abstract}

1. Artículo con enfoque cuantitativo resultado de un trabajo de grado en la modalidad de proyecto de investigación en el área de procesamiento digital de imágenes, desarrollado en el Grupo de Investigación en Control Avanzado - Gicav, y financiado por las Unidades Tecnológicas de Santander en la ciudad de Bucaramanga. Calle de los Estudiantes No. 9-82. PBX:6917700. Fecha de inicio: enero de 2015, fecha de terminación: mayo de 2016.

2. Ingeniero Electrónico, Unidades Tecnológicas de Santander. Estudiante de trabajo de grado en modalidad de proyecto de Investigación. Correo electrónico: diegorodriguez_978@hotmail.com. Investigador en formación, H-índex=0.

3. Ingeniero Electrónico, Unidades Tecnológicas de Santander. Estudiante de trabajo de grado en modalidad de Proyecto de Investigación. Investigador en formación, H-índex=0. Correo electrónico: jahirg@yahoo.com.

4. Ingeniero Electrónico, Universidad Industrial de Santander UIS. Candidato a Magister en Diseño, Gestión y Dirección de Proyectos, Centro Panamericano de Estudios Superiores Cepes. Docente- investigador del grupo: Gicav. Unidades Tecnológicas de Santander UTS de la ciudad de Bucaramanga (Colombia): Dirección: Calle de los Estudiantes n. ${ }^{\circ}$ 9-82, PBX: 6917700. Orcid: 0000-0003-3117-4081. Correo institucional: gicav@ correo.uts.edu.co.

5. Ingeniero Electrónico, Magíster en Ingeniería, Magíster en Procesamiento de imágenes, audio, señales y telecomunicaciones y PhD en Ingeniería Electrónica. Docente investigador en el grupo GPS de la Universidad de Investigación y Desarrollo. Orcid: 0000-0002-5377-8487. Correo institucional: esilva6@udi.edu.co. 
Palabras clave: sensor Kinect, modelos activos de forma ASM, PCA, OpenCV, reconocimiento de puntos característicos.

\begin{abstract}
This work shows the performance evaluation of a face tracking features algorithm, applying active shape models (ASM) and using the Kinect sensor as the capture image device. The development was implemented by using OpenCV libraries, in a laptop with processor core is at $2.4 \mathrm{GHz}, 4 \mathrm{~GB}$ of RAM, and Windows 7 operative system. In order to perform the evaluation, the algorithm was run to analyze the response under different facial expression poses. The stabilization times of the points over the image were measured and the localization of the points in the image was manually evaluated. The face was divided in regions: face contours, eyebrows, nose, eyes and mouth. Finally, the results are presented as the average time of the face matching, the average number of frames required to perform matching, and the average error of the positioning in different faces conditions. The results show the strength of this work and the adaptability for future work.
\end{abstract}

Key words: kinect sensor, active shape models ASM, PCA, OpenCV, feature points recognition.

El ajuste de un modelo deformable es el gran problema de registrar un modelo de forma parametrizada en una imagen de manera que su marcas correspondan a las ubicaciones consistentes en el objeto de interés (Cheng, Y., 1995). Este es un problema difícil, ya que implica una optimización en las dimensiones de altura, donde la apariencia puede variar mucho entre instancias del objeto debido a las condiciones de iluminación, ruido de la imagen, la resolución y las fuentes intrínsecas de variabilidad.

Se han propuesto muchos enfoques para esto con diversos grados de éxito (Cheng, Y., 1995; Cootes \& Taylor, 1992; Cristinacce \& Cootes, 2004, 2006, 2007). De ellos, uno de los más prometedores es el que utiliza una representación basada en parches (Baltrusaitis, Robinson \& Morency, 2012) en donde se tienen en cuenta las observaciones hechas en cada marcación de la imagen y su relación con las demás.

Este proyecto se desarrolló en una $\mathrm{PC}$, empleando el sensor Kinect como dispositivo de adquisición de imagen, sistema operativo Windows 7 de 32 Bits, el SDK Kinect for Windows, las librerías OpenCV, lenguaje de programación Python 2.7 de 32 Bits, y paquetes básicos para Python, entre los que se destacan el PyKinect, Numpy, Scipy, Cython y PyQT. Como IDE se utiliza el programa Geany y como GUI el paquete de PythonPyQt. Todo dentro de un ambiente de software de uso libre.

Las técnicas que se utilizaron para el seguimiento de las características faciales de forma activa son el reconocimiento de rostros, el PCA (Principal Component Analysis) (Delac, Grgic, \& Grgic, 2005), el PDM (Point Distribuid Model) (Chen \& Davoine, 2006; Viola \& Jones, 2001), CLM (Constrained Limited Model) (Baltrusaitis, Robinson \& Morency, 2012; Dornaika \& Davoine, 2004) y el modelo de parches (Baltrusaitis et al.; Cheng, S., Zafeiriou, Asthana, \& Pantic, 2014; Cristinacce \& Cootes, 2004).
El entrenamiento de dicho modelo se hizo con ayuda de la base de rostros MUTC desarrollada por la Universidad del Cabo, para uso investigativo, la cual contiene fotografías para cada individuo en diferentes condiciones de luminosidad y posición.

Mediante el paquete PyKinect y el SDK Kinect se tiene acceso al sensor para realzar la toma de muestra. Esta se procesa mediante el OpenCV, y se analiza con la matriz de entrenamiento, si hay resultado satisfactorio tendremos vector con las coordenadas de los puntos identificados, el cual es usado para graficar los resultados y permite hacer el cálculo de error.

\section{Metodología utilizada}

\section{Detección de rostro}

Una vez tomada la matriz de la imagen el primer paso es la detección de rostro, con lo cual se busca definir el ROI (Region of Interest) (Delac et al., 2005) y de esta forma continuar el análisis. Dado que es la búsqueda de un rostro y no la detección de un individuo en particular, OpenCV facilita esta tarea usando HaarCascades basadas en el algoritmo de Viola-Jones (Viola \& Jones, 2001).

\section{Restricción de los modelos locales}

La mayoría de los métodos de ajuste emplean una aproximación lineal de cómo su marco de objeto no rígido se deforma, esto da origen el PDM (modelo de distribución de puntos) (Cootes \& Taylor, 1992). El PDM modela las variaciones del marco no rígidas linealmente y la constituye con una transformación rígida global, ubicando el marco dentro de la imagen, como se muestra en la Ecuación 1.

$$
x_{i}=s R\left(x_{i}+\varphi_{i} q\right)+t
$$


Donde $x_{i}$ son las coordenadas de las marcas de la distribución de puntos i y el conjunto de valores $\{s, R, \varphi, t\}$ son los parámetros del PDM, los cuales son sescala global, $R$ rotación, $t$ traslación, y $\varphi$ un conjunto de parámetros no rígidos.

\section{Modelos locales limitados (CLM)}

Para evitar los inconvenientes de enfoques holísticos, tales como la complejidad del modelo y la sensibilidad a los cambios de iluminación, se usa un enfoque global que utiliza un conjunto de detectores (Cheng, Y., 1995; Cootes \& Taylor, 1992; Cristinacce \& Cootes, 2004, 2006, 2007; Wang, Lucey \& Cohn, 2008). Todas las instancias de CLMs buscan dos objetivos: (i) realizar una búsqueda local exhaustiva para cada marca del PDM en torno a su estimación actual utilizando algún tipo de detector de función, y (ii) optimizar los parámetros PDM de tal manera que la respuesta de detección sobre la totalidad de sus marcas de referencia sea conjuntamente máxima. La figura1, muestra el ajuste de los componentes CLM.
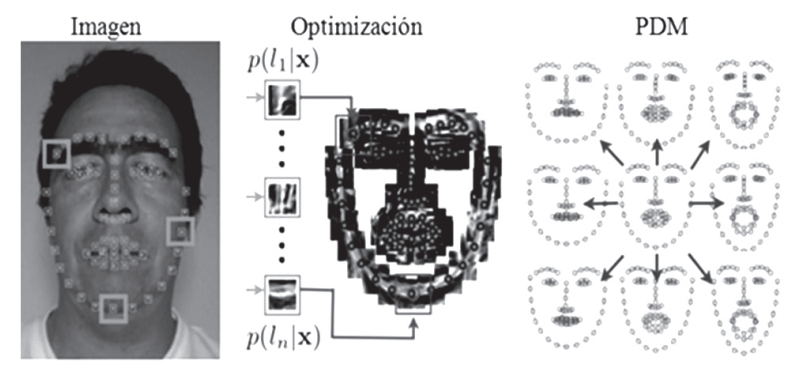

Figura 1.

Ajuste de CLM y sus componentes: (i) intensa búsqueda local de la ubicación de los rasgos para obtener el mapeo $\left\{p\left(l_{i}=\text { alineado } \mid l, x\right)\right\}_{(i=1)}^{n}$ y (ii) una respuesta de optimización para maximizar la respuesta PDM del ajuste de las marcas

\section{Intensa búsqueda local}

En la primera etapa de ajuste CLM (Baltrusaitis, Robinson \& Morency, 2012), se genera un mapa de probabilidad para cada marca de posición aplicando detectores locales para restringir las regiones alrededor de la estimación actual. Se ha propuesto un número de detectores de características para este propósito. Uno de los más sencillos, propuesto por Wang, Lucey y Cohn en Wang et al. (2008), es el regresor lineal logístico, que da el siguiente mapa respuesta para el i-ésimo punto de referencia obtenido a través de la ecuación 2.

$$
p\left(l_{i}=\text { alineado } \mid I, x\right)=\frac{1}{1+\exp \left\{\alpha C_{i}(I: x)+\beta\right\}}
$$

Donde $I_{i}$ es una variable aleatoria discreta que denota si la señal i-ésima está alineada correctamente o no. I es la imagen, $x$ es una ubicación 2D en la imagen, y $C i$ es un clasificador lineal dado por la Ecuación 3.

$$
C_{i}(I: x)=w_{i}^{T}\left[I\left(y_{1}\right) ; \ldots . . ; I\left(y_{m}\right)\right]+b_{i}
$$

Con $\left\{y_{i}\right\}_{i=1}^{m} \in \Omega_{x}$ (por ejemplo un parche de imagen). Una ventaja de usar este clasificador es que el mapa puede ser calculado usando eficientes operaciones de convolución.

\section{Optimización}

Una vez que se han encontrado los mapas de respuesta para cada lugar de interés, asumiendo la independencia condicional, se optimizan los procesos mediante la maximización con respecto a los parámetros $p$ del PDM, donde $x_{\text {}} i$ es parametrizado como en la Ecuación (1) y la dependencia de la imagen desciende para ser breve. Cabe señalar que algunas formas de CLMs plantean la Ecuación (4) minimizando la suma de las respuestas locales.

$$
\left.p\left(\left\{l_{i}=\text { anileado }\right\}_{i=n}^{n}\right) \mid P=\prod_{i=1}^{n} p\left(l_{i}=\text { alineado } \mid x_{i}\right) 4\right)
$$

El método simple utilizado por Cristinacce y Cootes (2004) es un optimizador genérico, sin embargo, la convergencia puede ser lenta cuando se utiliza para un PDM complejo con un gran número de parámetros.

\section{ASM}

La estrategia de optimización simple adecuada para CLM es la utilizada en el modelo de la forma activa (ASM) (Cootes \& Taylor, 1992). El método implica primero la búsqueda de la ubicación dentro de cada mapa respuesta para el cual se alcanzó el máximo valor dado por $\mu=\left[\mu_{1} ; \ldots ; \mu_{n}\right]$. El objetivo del procedimiento de optimización es, entonces, minimizar la diferencia ponderada de mínimos cuadrados entre el PDM y las coordenadas de las respuestas de los picos:

$$
Q(p)=\sum_{i=1}^{n} w_{i}\left\|x_{i}-\mu_{i}\right\|^{2}
$$

Donde los pesos $\left\{w_{i}\right\}_{i+1}^{n}$ reflejan la confianza sobre coordenadas de respuesta pico y se establecen normalmente a alguna función de las respuestas en $\left\{\mu_{i}\right\}_{i+1}^{n}$ haciéndolo más resistentes a cosas tales como la oclusión parcial, donde los puntos de referencia ocluidos se ponderan más débilmente.

La Ecuación (5) se minimiza de forma iterativa mediante el primer orden de expansión de Taylor de las marcas del PDM, tal como la Ecuación 6. 


$$
x_{i} \approx x_{i}^{c}+J_{i} \Delta p
$$

Resolviendo para la actualización de parámetros, siendo $\Delta \mathrm{p}$ la diferencia ponderada del mínimo cuadrado de las distancias:

$$
\Delta p=\left(\sum_{i=1}^{n} w_{i} J_{i}^{T} J_{i}\right)^{-1} \sum_{i=1}^{n} w_{i} J_{i}^{T}\left(\mu_{i}-x_{i}^{c}\right)
$$

Que luego se aplica de forma aditiva a los parámetros actuales: $p \leftarrow p+\Delta p$. Aquí, $J=\left[J_{1} ; \ldots J_{n}\right]$ es el Jacobiano y $\mathrm{x}^{\mathrm{c}}=\left[\mathrm{x}_{1} ; \ldots \mathrm{x}_{\mathrm{n}}\right]$ es el actual marco estimado.

Desde el punto de vista probabilístico introducido en CLM, el procedimiento de optimización de la ASM es equivalente a la aproximación de los mapas de respuesta con un estimador isotrópico de Gauss.

$$
p\left(l_{i}=\text { alineado } \mid x\right) \approx N\left(X ; \mu_{I}, \sigma_{i}^{2} I\right)
$$

Donde $w_{i}=\sigma_{i}^{2}$. Con esta aproximación, tomando el logaritmo negativo de la probabilidad en la ecuación (4) da como resultado el objetivo en la Ecuación (5). Es de aclarar que entre más complejo sea el marco y más cantidad de puntos tenga, el costo computacional se eleva, lo que se refleja en tiempos más grandes de procesamiento.

\section{Resultados}

En esta sección se describen los resultados de las pruebas realizadas para evaluar la eficiencia y el tiempo del algoritmo en el PC con sistema operativo Windows 7 32 bits con Procesador Core i5 @ 2.4Ghz, 4 Gigabytes de memoria RAM, y un disco de $256 \mathrm{~GB}$.

Para implementar las técnicas de reconocimiento hay dos cosas importantes: el entrenamiento y el algoritmo. Para realizar la detección se activa el sensor Kinect, se carga la imagen pasándola a escala de grises, y se aplica la detección de rostro por HAAR, lo cual determina el área de interés, ver Figura 3. En dicha región se hace la búsqueda del centro de los ojos y de la boca para hacer el análisis de posición, escalado, rotación y traslación. Ya con esto, se aplica el vector de entrenamiento para establecer las coincidencias de las marcas, posteriormente se aplica la optimización ASM, para así corregir posibles desviaciones por las oclusiones, ver Figura 2.

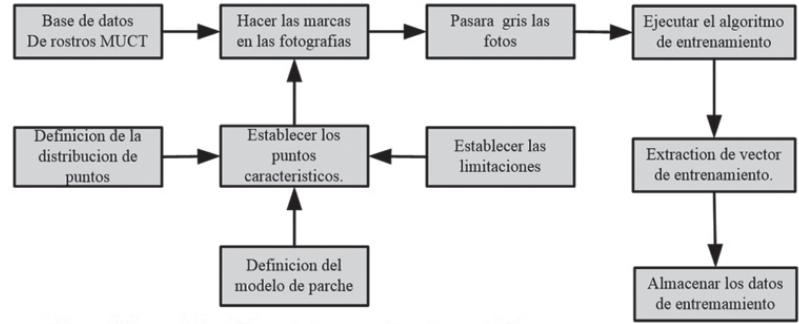

Figura 2.

Mapa de las etapas del proceso de entrenamiento

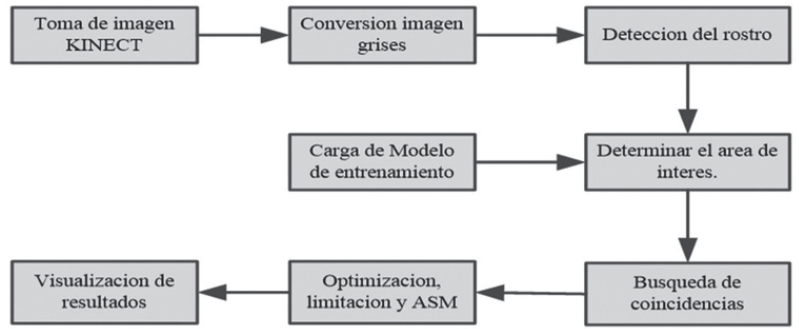

Figura 3.

Mapa de las etapas del proceso de reconocimiento

Dentro del aplicativo del proyecto es posible grabar video para su posterior análisis de error, o simplemente para visualizar su comportamiento en tiempo real; adicionalmente, se tiene la opción de manipular el motor para ajustar la inclinación del sensor Kinect.

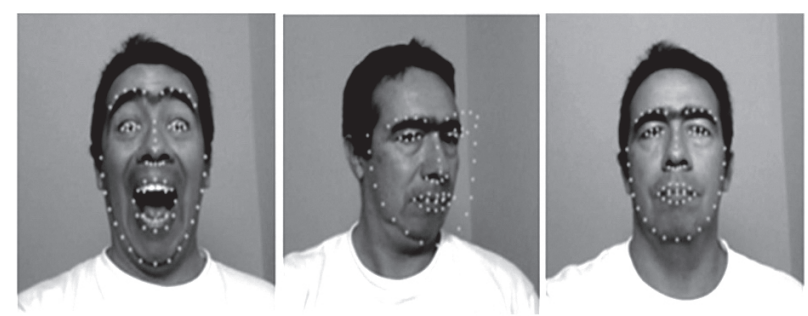

Figura 4.

Etapas del proceso de reconocimiento

Se realizaron pruebas para observar la respuesta del algoritmo respecto a las distintas posturas y expresiones faciales, ver Figura 4. Para esto se hizo correr el algoritmo diez veces sobre una postura inmóvil, al final, se midió el tiempo que tomó estabilizar los puntos sobre la imagen, y sobre la imagen final se analizó punto a punto y con criterio humano donde deberían estar localizados los puntos.

Para facilitar el análisis se agrupan los puntos acordes a la zona del rostro: contorno de la cara, cejas, nariz, ojos y boca, e igualmente se grafica el error punto a punto.

Ya con estos datos se calcula el error de ubicación. A continuación, se observan las distintas posturas y las tablas de resultados. 


\section{Postura cara frontal}

En la Figura 5 se muestra la marcación de los puntos sobre la postura cara frontal. Los resultados del cálculo del error de ubicación, presentados en la Tabla 1, muestran un seguimiento bastante real de la localización de los puntos, encontrándose los puntos más desviados en la parte inferior de la mandíbula.

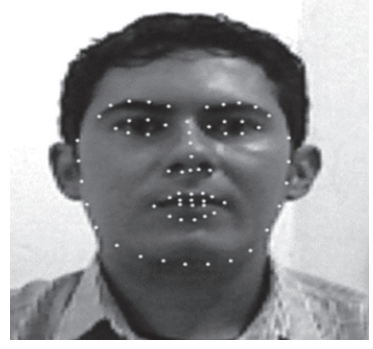

Figura 5.

Puntos encontrados para una cara frontal, una vez estabilizado

Tabla 1

Resumen de tiempos de estabilización y cálculo de error de ubicación del punto sobre la imagen de cara frontal neutra

\begin{tabular}{|c|c|c|c|c|c|c|c|}
\hline \multicolumn{8}{|c|}{ Análisis de resultados cara frontal } \\
\hline \multirow[b]{2}{*}{ Iteración } & \multicolumn{3}{|l|}{ Tiempo (s) } & \multicolumn{4}{|c|}{ Error RMS } \\
\hline & Estabilización & Contorno & Cejas & Nariz & Ojos & Boca & Global \\
\hline 1 & 1,462 & 6,32 & 5,41 & 3,12 & 5,83 & 4,77 & 5,24 \\
\hline 2 & 1,655 & 6,51 & 5,70 & 5,58 & 6,57 & 7,84 & 6,63 \\
\hline 3 & 1,888 & 5,85 & 7,09 & 4,43 & 6,28 & 6,75 & 6,17 \\
\hline 4 & 1,654 & 6,88 & 5,86 & 3,84 & 7,28 & 8,04 & 6,70 \\
\hline 5 & 1,914 & 7,45 & 6,60 & 4,69 & 6,98 & 6,97 & 6,73 \\
\hline 6 & 1,856 & 8,71 & 5,65 & 3,53 & 6,63 & 4,12 & 5,91 \\
\hline 7 & 2,018 & 6,86 & 7,06 & 3,07 & 7,86 & 5,09 & 6,07 \\
\hline 8 & 1,649 & 9,32 & 7,47 & 4,29 & 8,27 & 5,96 & 7,25 \\
\hline 9 & 1,831 & 6,92 & 7,15 & 4,76 & 8,26 & 6,71 & 6,85 \\
\hline 10 & 1,626 & 6,34 & 6,74 & 4,10 & 7,12 & 8,48 & 6,82 \\
\hline Promedio & 1,755 & 7,12 & 6,47 & 4,14 & 7,11 & 6,47 & 6,43 \\
\hline
\end{tabular}

Fuente: Autores

\section{Postura boca abierta}

La Figura 6 muestra la marcación de los puntos sobre el rostro frontal con la boca abierta. Los resultados del cálculo del error de ubicación, presentados en la Tabla 2, muestran un seguimiento bastante real de la localización de los puntos, notándose una desviación en general un poco mayor que en el rostro frontal neutro. La boca abierta permite demarcar mejor los puntos.

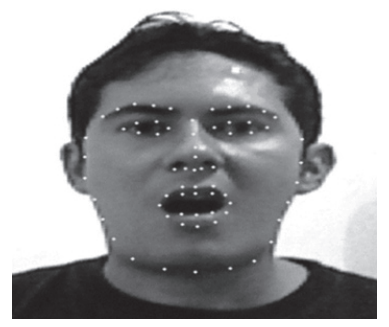

Figura 6.

Puntos encontrados para una cara frontal con la boca abierta, una vez estabilizado

Tabla 2

Resumen de tiempos de estabilización y cálculo de error de ubicación del punto sobre la imagen de cara frontal con boca abierta

\begin{tabular}{llcccccc}
\hline \multicolumn{7}{c}{ Análisis de resultados cara frontal boca abierta } \\
\hline \multirow{2}{*}{ Iteración } & \multicolumn{7}{c}{ Error RMS } \\
\cline { 2 - 8 } & Estabilización & Contorno & Cejas & Nariz & Ojos & Boca & Global \\
\hline 1 & 1,801 & 9,07 & 4,76 & 3,10 & 6,43 & 5,47 & 6,14 \\
2 & 1,685 & 8,41 & 5,65 & 10,33 & 7,74 & 5,35 & 7,30 \\
3 & 1,805 & 8,54 & 5,47 & 2,94 & 6,97 & 5,67 & 6,24 \\
4 & 1,642 & 7,52 & 6,04 & 4,16 & 8,55 & 6,07 & 6,63 \\
5 & 1,679 & 7,88 & 5,91 & 3,91 & 9,21 & 6,24 & 6,83 \\
6 & 1,724 & 6,63 & 5,88 & 3,12 & 16,96 & 6,11 & 7,77 \\
7 & 1,822 & 6,51 & 8,07 & 3,47 & 7,99 & 5,60 & 6,35 \\
8 & 1,839 & 8,20 & 5,38 & 4,53 & 7,58 & 9,96 & 7,64 \\
9 & 2,012 & 28,91 & 6,23 & 4,09 & 7,00 & 14,86 & 14,27 \\
10 & 1,846 & 9,39 & 4,74 & 4,10 & 7,58 & 5,93 & 6,69 \\
\hline Promedio & $\mathbf{1 , 7 8 5}$ & $\mathbf{1 0 , 1 1}$ & $\mathbf{5 , 8 1}$ & $\mathbf{4 , 3 8}$ & $\mathbf{8 , 6 0}$ & $\mathbf{7 , 1 3}$ & $\mathbf{7 , 5 9}$ \\
\hline
\end{tabular}

Fuente: Autores 


\section{Postura de sorpresa}

La Figura 7 muestra la marcación de los puntos sobre el rostro con gesto de sorpresa. Los resultados del cálculo del error de ubicación, con rostro frontal con sorpresa presentados en laTabla 3 continúan siendo muy acertados en cuanto a la localización de los puntos, e inclusive, la desviación es menor a la muestra de solo boca abierta; las cejas levantadas no representan problema, siendo estos puntos muy ajustados a la realidad.

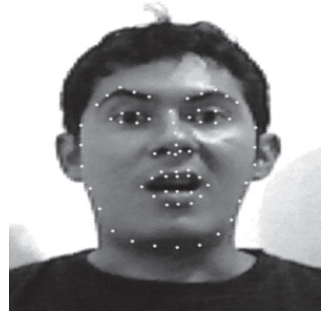

Figura 7.

Puntos encontrados para una expresión de sorpresa, una vez estabilizado

Tabla 3

Resumen de tiempos de estabilización y cálculo de error de ubicación del punto sobre la imagen de cara frontal sorpresa

\begin{tabular}{|c|c|c|c|c|c|c|c|}
\hline \multicolumn{8}{|c|}{ Análisis de resultados cara frontal sorpresa } \\
\hline \multirow[b]{2}{*}{ Iteración } & \multicolumn{3}{|c|}{ Tiempo (s) } & \multicolumn{4}{|c|}{ Error RMS } \\
\hline & Estabilización & Contorno & Cejas & Nariz & Ojos & Boca & Global \\
\hline 1 & 1,804 & 8,51 & 4,17 & 5,32 & 5,36 & 9,61 & 7,15 \\
\hline 2 & 1,713 & 7,80 & 3,67 & 3,67 & 5,97 & 5,32 & 5,60 \\
\hline 3 & 1,685 & 6,41 & 5,40 & 5,19 & 7,00 & 8,08 & 6,65 \\
\hline 4 & 1,805 & 6,32 & 4,36 & 4,28 & 4,92 & 7,28 & 5,75 \\
\hline 5 & 1,830 & 9,74 & 4,25 & 5,51 & 6,04 & 10,53 & 7,88 \\
\hline 6 & 1,830 & 10,11 & 4,87 & 4,97 & 5,17 & 8,40 & 7,25 \\
\hline 7 & 2,030 & 8,78 & 3,75 & 4,90 & 5,84 & 6,81 & 6,42 \\
\hline 8 & 1,462 & 9,00 & 5,77 & 5,78 & 6,19 & 8,54 & 7,44 \\
\hline 9 & 2,179 & 19,79 & 6,54 & 5,34 & 5,98 & 6,38 & 9,64 \\
\hline 10 & 1,459 & 6,49 & 5,58 & 4,62 & 5,97 & 5,92 & 5,85 \\
\hline Promedio & 1,779 & 9,30 & 4,84 & 4,96 & 5,84 & 7,69 & 6,96 \\
\hline
\end{tabular}

Fuente: Autores

\section{Postura cara hacia arriba}

La Figura 8 muestra la marcación de los puntos sobre el rostro con postura cara hacia arriba. En la Tabla 4 se presentan los resultados, los cuales siguen siendo acertados en la localización de los puntos; no obstante, para esta escena hay un incremento en desviaciones de los puntos del borde inferior de la mandíbula y de la nariz.

Tabla 4

Resumen de tiempos de estabilización y cálculo de error de ubicación del punto sobre la imagen de cara hacia arriba

\begin{tabular}{|c|c|c|c|c|c|c|c|}
\hline \multicolumn{8}{|c|}{ Análisis de resultados cara hacia arriba } \\
\hline \multirow[b]{2}{*}{ Iteración } & \multicolumn{3}{|l|}{ Tiempo (s) } & \multicolumn{4}{|c|}{ Error RMS } \\
\hline & Estabilización & Contorno & Cejas & Nariz & Ojos & Boca & Global \\
\hline 1 & 1,061 & 6,60 & 8,40 & 4,23 & 5,34 & 2,46 & 5,19 \\
\hline 2 & 1,268 & 12,79 & 8,00 & 4,82 & 7,02 & 2,54 & 7,13 \\
\hline 3 & 1,392 & 24,46 & 7,62 & 29,42 & 5,37 & 4,87 & 13,77 \\
\hline 4 & 1,924 & 9,63 & 7,80 & 4,33 & 10,86 & 3,35 & 7,14 \\
\hline 5 & 2,950 & 17,40 & 7,09 & 33,48 & 5,76 & 5,56 & 12,69 \\
\hline 7 & 1,440 & 8,75 & 6,45 & 3,98 & 6,99 & 3,31 & 5,95 \\
\hline 8 & 1,281 & 12,47 & 6,86 & 4,25 & 6,83 & 3,50 & 7,03 \\
\hline 9 & 1,564 & 12,21 & 6,51 & 2,97 & 7,43 & 3,88 & 6,95 \\
\hline 10 & 1,396 & 7,97 & 6,96 & 4,14 & 8,23 & 3,79 & 6,20 \\
\hline Promedio & 1,565 & 11,84 & 6,99 & 9,53 & 7,01 & 3,59 & 7,66 \\
\hline
\end{tabular}

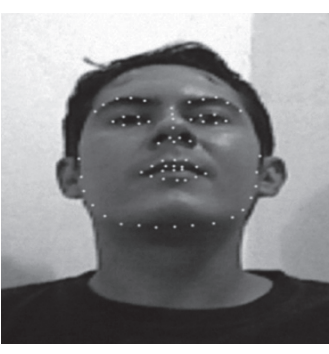

Figura 8.

Puntos encontrados en un rostro inclinando la cabeza hacia atrás, una vez estabilizado

Fuente: Autores 


\section{Postura rostro inclinado a la izquierda}

La Figura 9 muestra la marcación de los puntos sobre el rostro con postura de cara inclinada a la izquierda. En la Tabla 5 se presentan los resultados del cálculo del error de ubicación, los cuales continúan siendo muy satisfactorios, pues su error es muy similar al obtenido con un rostro frontal neutro.

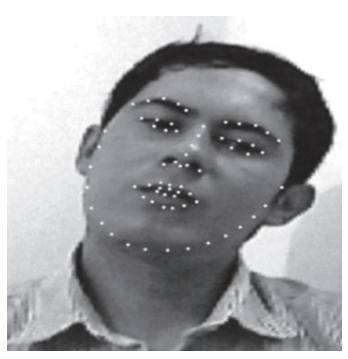

Figura 9.

Puntos encontrados en un rostro inclinado a la izquierda, una vez estabilizado

Tabla 5

Resumen de tiempos de estabilización y cálculo de error de ubicación del punto sobre la imagen de cara inclinada a la izquierda

\begin{tabular}{|c|c|c|c|c|c|c|c|}
\hline \multicolumn{8}{|c|}{ Análisis de resultados cara hacia la izquierda } \\
\hline \multirow[b]{2}{*}{ Iteración } & \multicolumn{3}{|c|}{ Tiempo (s) } & \multicolumn{4}{|c|}{ Error RMS } \\
\hline & Estabilización & Contorno & Cejas & Nariz & Ojos & Boca & Global \\
\hline 1 & 1,221 & 10,48 & 5,61 & 5,34 & 7,33 & 3,11 & 6,46 \\
\hline 2 & 1,576 & 16,25 & 5,47 & 4,82 & 6,13 & 3,69 & 7,79 \\
\hline 3 & 1,529 & 6,45 & 4,78 & 5,64 & 5,50 & 3,09 & 5,00 \\
\hline 4 & 1,202 & 11,20 & 4,31 & 6,53 & 4,91 & 3,51 & 6,28 \\
\hline 5 & 1,170 & 7,38 & 2,98 & 6,30 & 5,23 & 4,04 & 5,26 \\
\hline 7 & 1,373 & 6,69 & 4,25 & 6,88 & 5,32 & 3,05 & 5,10 \\
\hline 8 & 1,358 & 11,31 & 3,94 & 6,87 & 5,34 & 2,69 & 6,15 \\
\hline 9 & 1,528 & 9,37 & 4,92 & 5,98 & 5,47 & 3,33 & 5,87 \\
\hline 10 & 1,404 & 13,05 & 5,03 & 5,58 & 5,25 & 3,35 & 6,75 \\
\hline Promedio & 1,371 & 10,38 & 4,38 & 5,97 & 5,61 & 3,42 & 6,10 \\
\hline
\end{tabular}

Fuente: Autores

\section{Postura rostro inclinado a la derecha}

La Figura 10 muestra la marcación de los puntos sobre el rostro con postura de cara inclinada a la derecha. En la Tabla 6 se presentan los resultados del cálculo del error de ubicación, los cuales son muy similares a una inclinación a la izquierda y frontal neutro, pues inclinar el rostro no representa desviaciones representativas.

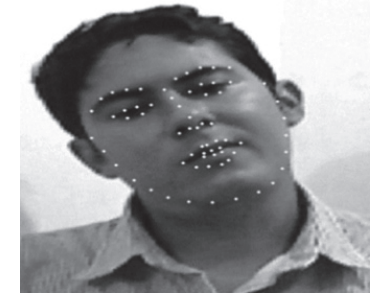

Figura 10.

Puntos encontrados en un rostro inclinado a la derecha, una vez estabilizado

Tabla 6

Resumen de tiempos de estabilización y cálculo de error de ubicación del punto sobre la imagen de cara inclinada a la derecha

\begin{tabular}{llcccccc}
\hline \multicolumn{7}{c}{ Análisis de resultados cara hacia la derecha } \\
\hline \multirow{2}{*}{ Iteración } & \multicolumn{5}{c}{ Error RMS } \\
\cline { 2 - 7 } & Estabilización & Contorno & Cejas & Nariz & Ojos & Boca & Global \\
\hline 1 & 1,247 & 5,51 & 6,42 & 6,50 & 6,46 & 4,85 & 5,87 \\
2 & 1,286 & 6,94 & 5,82 & 5,20 & 6,62 & 4,08 & 5,69 \\
3 & 1,251 & 9,08 & 3,02 & 4,90 & 6,63 & 4,40 & 5,87 \\
4 & 1,767 & 5,24 & 5,73 & 5,31 & 6,17 & 6,27 & 5,77 \\
5 & 1,943 & 19,34 & 3,70 & 5,24 & 6,31 & 3,95 & 8,48 \\
6 & 1,246 & 9,62 & 4,13 & 3,35 & 5,87 & 3,90 & 5,69 \\
7 & 1,389 & 12,07 & 15,96 & 5,92 & 8,17 & 6,77 & 9,67 \\
8 & 1,415 & 7,02 & 7,96 & 15,32 & 8,14 & 5,14 & 7,98 \\
9 & 1,300 & 10,33 & 5,60 & 4,54 & 7,10 & 4,96 & 6,77 \\
10 & 0,896 & 5,43 & 4,38 & 4,18 & 6,30 & 4,94 & 5,13 \\
\hline Promedio & $\mathbf{1 , 3 7 4}$ & $\mathbf{9 , 0 6}$ & $\mathbf{6 , 2 7}$ & $\mathbf{6 , 0 5}$ & $\mathbf{6 , 7 8}$ & $\mathbf{4 , 9 3}$ & $\mathbf{6 , 6 9}$ \\
\hline
\end{tabular}

Fuente: Autores 


\section{Conclusiones}

Al culminar esta investigación acerca del desempeño del algoritmo de seguimiento de las características faciales con respecto a las distintas posturas y expresiones faciales se comprobó que el algoritmo emplea un tiempo promedio de ajuste del modelo menor a 2 segundos y un promedio de frames menor a 9. Además, que el contorno del rostro es el que mayor porcentaje de imprecisión presenta.

En condiciones de luminosidad favorables el desempeño del algoritmo es bastante bueno, sin embargo, existen posturas del rostro como girar la cabeza sobre el eje vertical en donde es común que cometa errores con mayor frecuencia. Igualmente, si la persona hace gestos muy rápidos como gesticular con la boca.

Para inicializar el modelo se debe empezar con una postura natural normal, es decir con el rostro de forma frontal y sin inclinaciones; una vez detectado el rostro, el modelo lo puede mover a posiciones muy cercanas a los noventa grados de inclinación, es decir casi en posición horizontal.

En términos generales el algoritmo trabaja correctamente, aunque con limitaciones de posturas. Sin embargo, durante las pruebas se detectó que si el fondo es muy ruidoso o hay múltiples sombras y objetos, es posible tener un falso positivo que hace que el algoritmo encuentre una región y haga marcación de los puntos en la imagen.

A través de la realización de este proyecto se pudo determinar que la creación de un algoritmo de seguimiento a las características faciales es un campo en continuo desarrollo. Si bien existen muchos enfoques, estos deben ser optimizados dado que el coste computacional debe ser bajo; de lo contrario, su uso en tiempo real se verá muy limitado.

A futuro este proyecto se puede optimizar para realizar diferentes trabajos en el seguimiento de expresiones faciales en tiempo real con dispositivos de bajo costo y más pequeños que el sensor Kinect, pudiéndose aplicar a sistemas embebidos como el Rapsberry Pi, que tengan una mejor eficiencia energética y menores requisitos computacionales.

\section{Referencias}

Baltrusaitis, T., Robinson, P. \& Morency, L. (2012). 3D constrained local model for rigid and non-rigid facial tracking. Computer Vision and Pattern Recognition (CVPR), 2012
IEEE Conference on (pp. 2610-2617). IEEE.

Chen, Y. \& Davoine, F. (2006). Simultaneous Tracking of Rigid Head Motion and Non-rigid Facial Animation by Analyzing Local Features Statistically. BMVC, 609-618.

Cheng, S., Zafeiriou, S., Asthana, A. \& Pantic, M. (2014). 3D facial geometric features for constrained local mode. IEEE, 14251429.

Cheng, Y. (1995). Mean shift, mode seeking, and clustering. IEEE, 17(8), 790-799.

Cootes, F. \& Taylor, J. (1992). Active shape models - "smart snakes". Springer, BMVC92, 266-275.

Cristinacce, D. \& Cootes, F. (2004). A comparison of shape constrained facial feature detectors. IEEE, 375-380.

Cristinacce, D. \& Cootes, F. (2006). Feature Detection and Tracking with Constrained Local Models. BMVC, 10.

Cristinacce, D. \& Cootes, F. (2007). Boosted regression active shape models. BMVC, 7.

Delac, K., Grgic, M. \& Grgic, S. (2005). Independent comparative study of PCA, ICA, and LDA on the FERET data set. International Journal of Imaging Systems and Technology, 15(5), 252-260.

Dornaika, F. \& Davoine, F. (2004). Head and facial animation tracking using appearance-adaptive models and particle filters. IEEE, 153-153.

Viola, P. \& Jones, M. (2001). Rapid object detection using a boosted cascade of simple features. IEEE, I-511.

Wang, Y., Lucey, S. \& Cohn, F. (2008). Enforcing convexity for improved alignment with constrained local models. CVPR 2008. IEEE Conference on (pp. 1-8). IEEE. 